\title{
Labyrinthe
}

3 | 1999

Numéro 3

\section{Ouverture mécanique de la double hélice de l'ADN}

\section{Baptiste Essevaz-Roulet}

\section{(2) OpenEdition}

Journals

Édition électronique

URL : http://journals.openedition.org/labyrinthe/66

DOI : $10.4000 /$ labyrinthe.66

ISSN : 1950-6031

Éditeur

Hermann

Édition imprimée

Date de publication : 15 avril 1999

Pagination : 119-121

Référence électronique

Baptiste Essevaz-Roulet, « Ouverture mécanique de la double hélice de l'ADN », Labyrinthe [En ligne], 3 | 1999, mis en ligne le 07 février 2005, consulté le 22 septembre 2020. URL : http://

journals.openedition.org/labyrinthe/66; DOI : https://doi.org/10.4000/labyrinthe.66

Ce document a été généré automatiquement le 22 septembre 2020.

Propriété intellectuelle 


\title{
Ouverture mécanique de la double hélice de l'ADN
}

\author{
Baptiste Essevaz-Roulet
}

1 Quel est le point commun à tous les êtres vivants sur terre ? Leur capacité à se reproduire est généralement considérée comme la définition de la vie. Cependant, la seule reproduction ne suffit pas à perpétuer une espèce : la descendance doit être identique -ou presque- aux géniteurs... Un chat ne donne pas un chien. Il y a environ un demi-siècle, les scientifiques ont pu mettre un nom sur ce qui permettait la reproduction à l'identique : l'ADN. Cette molécule, dont les initiales signifient Acide DésoxyriboNucléique, contient toutes les informations nécessaires à la construction d'un être vivant. Cette mémoire, dite patrimoine génétique, est contenue dans la structure même de la molécule, un peu à la manière d'un livre dont chaque page aurait la forme d'une lettre. Chaque espèce a un patrimoine propre, différent de celui des autres espèces, et, dans une moindre mesure, le patrimoine de chaque individu d'une même espèce diffère légèrement de celui de son congénère. C'est ce qui vous permet de ne pas être exactement comme votre voisin.

2 Les molécules d'ADN sont localisées dans le noyau de chaque cellule vivante (de l'éponge à l'homme en passant par la tulipe et le cornichon). A quoi ressemble une molécule d'ADN ? Elle est constituée de deux brins complémentaires qui s'enroulent l'un autour de l'autre en double hélice. Sa longueur est très grande devant son épaisseur : sa largeur est de 2 nanomètres $(1$ nanomètre $=$ un millionième de millimètre), alors que sa longueur, qui varie d'une espèce à l'autre, peut atteindre 2 mètres chez l'homme. Proportionnellement, c'est comme une ficelle de $1 \mathrm{~mm}$ de diamètre pour 1000 kilomètres de long ! D'autre part, si chacun des brins est complémentaire l'un de l'autre, c'est parce que chaque brin porte un enchaînement de groupements chimiques en série qui a son exact complémentaire sur l'autre brin. Ces groupements chimiques sont appelés « bases » et il en existe 4 sortes : A, T, G et C. Ces bases marchent par paire : A est complémentaire de T, et $\mathrm{G}$ de $\mathrm{C}$. Ainsi, lorsqu'un brin porte une série AGGCTTA par exemple, le brin complémentaire portera la série TCCGAAT. Ce genre de série est appelé la "séquence ", et parfois "la séquence 
génomique » parce que c'est elle qui contient l'infor-mation génétique. Il y a environ 3 millions de bases par millimètre d'ADN.

3 Les biologistes s'intéressent de près aux interactions entre ces deux brins pour comprendre à terme la véritable machinerie moléculaire de la cellule qui fonctionne autour de l'ADN. Les généticiens, eux, veulent décrypter la séquence de l'ADN pour identifier d'abord, guérir ensuite, les maladies génétiques qui sont souvent dues à une erreur dans la séquence. De nombreuses techniques, généralement lourdes et onéreuses existent pour étudier la molécule. Elles sont performantes mais donnent des informations partielles, très indirectes, correspondant à des moyennes sur des millions de molécules. C'est pourquoi les physiciens, en se penchant sur le problème, se sont intéressés à des expériences qui permettraient de travailler sur des molécules uniques. C'est une démarche qui date d'une dizaine d'années seulement et qui est possible grâce aux progrès techniques en matière de biochimie moléculaire et micromanipulation. On a vu, par exemple, des équipes de physiciens tripoter l'ADN dans tous les sens : traction, torsion et autres persécutions. Parmi ces équipes, plusieurs se situent à l'Institut Curie et à l'Ecole Normale Supérieure à Paris. C'est dans ce contexte que nous avons désiré faire subir à l'ADN l'ultime torture : l'écartèlement, ou plutôt, l'ouverture forcée mécaniquement de la double hélice.

Comment ouvrir une unique molécule d'ADN ? Il faut d'abord réussir à isoler une seule molécule parmi des millions et attraper chacun de ses brins indépendamment. Une solution d'ADN est spécialement préparée à cet effet. Chaque molécule de cette solution a un de ses deux brins capable de se greffer sur une bille microscopique, alors que l'autre brin se collera sur une surface de verre. Ainsi, chacune des molécules est reliée à une seule bille, ce qui permet de les isoler sous microscope pour travailler sur la molécule de son choix. Enfin, pour attraper la molécule, une canne à pêche en verre aussi fine qu'un fil de toile d'araignée est collée sur la bille. L'expérience peut alors commencer : la lamelle de verre sur laquelle est greffé un des brins de l'ADN est déplacée très lentement, alors que la base de la fibre de verre reste fixe (voir figure 1). À mesure que la surface s'éloigne du point de départ, l'extrémité de la fibre est petit à petit défléchit. C'est cette déflexion qui nous renseigne sur la force que nous exerçons sur la molécule.

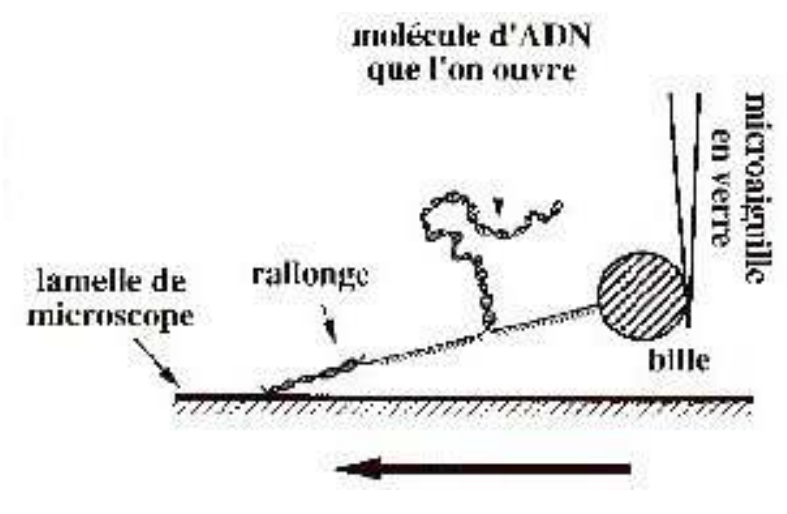

Fig. 1 : Principe du dispositif expérimental

La surface est déplacée tandis que la base de la microaiguille reste fixe.

5 Que constate-t-on ? La force subie par la molécule augmente de plus en plus, jusqu'à ce qu'elle soit suffisante pour provoquer la rupture des premières paires de bases de l'ADN. La force de rupture est alors de 10 picoNewton environ, soit une force un million 
de fois plus petite que celle qu'exercerait la Terre sur une masse d'un milligramme. C'est tout petit, mais pas négligeable du tout, car beaucoup de processus biochimiques développent des forces de cet ordre de grandeur dans la cellule. Lorsqu'on continue à déplacer la surface, la force présente de petites fluctuations : elle monte lentement puis chute brutalement (voir figure 2). Comment interpréter ces « dents de scie »? Elles sont liées à la séquence de la molécule. En effet, les paires de bases G-C sont plus solides que les paires de bases A-T. Dans les zones « dures ", riches en G-C, l'ouverture est bloquée ; comme la surface se déplace toujours, la force monte. Lorsque la force a atteint une valeur seuil, l'ou-verture de plusieurs paires de bases a lieu d'un seul coup et la force chute. Ainsi, l'ouverture se produit par saccades. Sur la figure 2, le signal de la force est superposé à la séquence. On remarque effectivement que les montées en force correspondent aux zones riches en G-C.

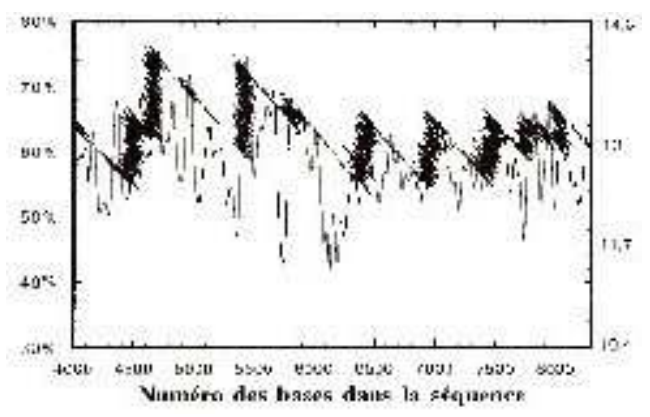

Fig. 2 : Signal de force superposée à la séquence

En trait continu : pourcentage en paires de bases G-C de la séquence

En petits points : force exercée sur la molécule (en PicoNewton)

6 Nous avons réalisé pour la première fois l'ouverture de l'ADN et mesuré la force qui lie les deux brins. Nous avons également montré que le signal de force est corrélé à la séquence de la molécule. Par ailleurs, la molécule s'est révélée être d'une surprenante robustesse : on peut, par exemple refermer la molécule après l'avoir ouverte, puis la rouvrir autant de fois que l'on veut avec un signal toujours reproductible, pour peu que l'on procède avec délicatesse... On rentre ainsi dans l'intimité d'une molécule d'ADN, en la dégrafant progressivement, puis en regardant à l'intérieur... Erotique, non? 